\title{
Development and external validation of a prognostic nomogram for acute decompensation of chronic hepatitis B cirrhosis
}

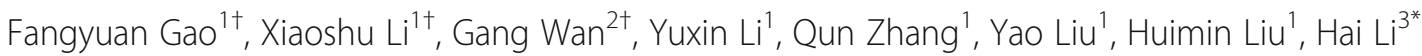
and Xianbo Wang ${ }^{1 *}$ (D)

\begin{abstract}
Background: Acute decompensation (AD) has been shown to be associated with a high mortality rate for cirrhosis patients. This study aimed to develop a prognostic nomogram to evaluating the individual prognosis for AD of cirrhosis in chronic hepatitis B (CHB).

Methods: The nomogram was developed using data from a retrospective study on 509 patients hospitalized for AD of CHB cirrhosis from October 2008 to February 2014 at the Beijing Ditan Hospital, Capital Medical University. The predictive accuracy, discriminative ability, and clinical net benefit were evaluated by concordance index (C-index), calibration curves, and decision curve analysis (DCA). The results were validated on 620 patients consecutively enrolled from January 2005 to December 2010 at the Renji Hospital, Shanghai Jiao Tong University,.

Results: On multivariate analysis of the derivation cohort, independent factors included in the nomogram were age, previous decompensation, bacterial infection, hepatic encephalopathy, and total bilirubin. The calibration curve for the probability of survival showed good agreement between the nomogram and actual observation. The nomogram had a C-index of 0.897, which was statistically higher than the C-index values of CTP (0.793), MELD (0. 821), SOFA (0.868), or the Chronic Liver Failure Consortium AD (CLIF-C AD) (0.716) scores $(p<0.001$ for all). Using DCA, the nomogram also demonstrated superior net benefits over other score models. The results were confirmed in the validation cohort.
\end{abstract}

Conclusions: The proposed nomogram enables more-accurate individualized prediction of survival than MELD, CTP, SOFA, or CLIF-C AD scores for AD of CHB cirrhosis patients.

Keywords: Chronic liver disease, Chronic hepatitis B, Nomogram, Prognosis

\section{Background}

Hepatitis B virus (HBV) infection is a critical global health threat, particularly in Asia. In the natural history of chronic HBV infection, partial patients remain asymptomatic until an episode of acute decompensation (AD), which is featured by the rapid development of one or

\footnotetext{
* Correspondence: wangxb@ccmu.edu.cn; haili_17@126.com

${ }^{\dagger}$ Fangyuan Gao, Xiaoshu Li and Gang Wan contributed equally to this work.

${ }^{3}$ Department of Gastroenterology, Ren Ji Hospital, School of Medicine,

Shanghai Jiao Tong University, Shanghai, China

${ }^{1}$ Center of Integrative Medicine, Beijing Ditan Hospital, Capital Medical

University, Beijing, China

Full list of author information is available at the end of the article
}

more major complications of liver disease. $\mathrm{AD}$ is the common cause of hospital admission and is associated with a high mortality rate for cirrhosis patients. AD of cirrhosis occurs in as many as $15 \%$ of cirrhosis patients each year, and the frequency and severity will increase with the progressively deterioration of liver reserve function. The course of illness during a patient's early hospital phase has been related to eventual prognosis. Therefore, early recognition and aggressive treatment are important to improving survival [1-4].

Recently, the CANONIC research team built the Chronic Liver Failure Consortium AD (CLIF-C AD),

(c) The Author(s). 2018 Open Access This article is distributed under the terms of the Creative Commons Attribution 4.0 International License (http://creativecommons.org/licenses/by/4.0/), which permits unrestricted use, distribution, and reproduction in any medium, provided you give appropriate credit to the original author(s) and the source, provide a link to the Creative Commons license, and indicate if changes were made. The Creative Commons Public Domain Dedication waiver (http://creativecommons.org/publicdomain/zero/1.0/) applies to the data made available in this article, unless otherwise stated. 
which was demonstrated to be superior to the Model for End-stage Liver Disease (MELD), MELD-Na, and Child-Turcotte-Pugh (CTP) assessments in predicting mortality due to $\mathrm{AD}$ [5]. However, the current score models were built primarily base on populations with alcoholism issues and/or hepatitis $C$ virus infections [5]. To date, no reports of a model have been found to assess the prognosis of $\mathrm{AD}$ of chronic hepatitis $\mathrm{B}(\mathrm{CHB})$ cirrhosis. $\mathrm{CHB}$ cirrhosis is deemed as the leading cause of $\mathrm{AD}$ in the Asia-Pacific region [6]. Therefore, it is necessary to create a risk model that can predict the prognosis for $\mathrm{AD}$ of $\mathrm{CHB}$ cirrhosis.

The nomogram is a graphical calculating and two-dimensional diagram, which could offer more precise and individualized prediction relative to the traditional models for multiple illness [6-9]. The specific objective of the study was to determine the risk factors for short-term death of $\mathrm{AD}$ with $\mathrm{CHB}$ cirrhosis, develop a prognostic nomogram to estimate the individual prognosis, and promote the implementation of preventive measures.

\section{Patients and methods Derivation cohort}

In total, 1781 patients hospitalized at Beijing Ditan Hospital (Beijing, China), which is affiliated with the Capital Medical University, between October 2008 and February 2014 with $\mathrm{CHB}$ cirrhosis were screened, and 509 patients with $\mathrm{AD}$ of $\mathrm{CHB}$ cirrhosis were enrolled for model derivation. All patients were limited to age $\geq 18$ years in this study, and patients with the following diseases would be excluded: (1) infected with hepatitis virus (including A, C, D, and E), human immunodeficiency virus, or other viruses; (2) autoimmune hepatitis, drug-induced liver injury, alcohol-induced liver disease, Wilson's disease, liver cancer, or hemolytic diseases; (3) other fatal disease, or gestation; or (4) liver operation or liver transplantation. The Ethics Committee from Beijing Ditan Hospital has approved this study protocol.

\section{Validation cohort}

2610 patients hospitalized with CHB cirrhosis at Renji Hospital, Shanghai Jiao Tong University, from January 2005 to December 2010 were screened, and 620 patients with $\mathrm{AD}$ of $\mathrm{CHB}$ cirrhosis were enrolled for external validation of the model. Only patients meeting the inclusion and exclusion standard and having sufficient data were registered. The research was supported by the Ethics Committee from Renji Hospital.

Cirrhosis was confirmed by previous hepatic pathology or clinical signs and results of laboratory detection, radiologic and endoscopic presentation.

$\mathrm{AD}$ of cirrhosis was defined as the rapid development of one or more major complications (ie, large ascites, hepatic encephalopathy (HE), gastrointestinal hemorrhage and bacterial infection) of liver disease [10-14]. Cirrhosis patients with grade 2 to 3 ascites within 2 weeks could be diagnosed with the acute development of large ascites [10]. Acute HE was characterized by the development of the acute confusional status in patients with previously normal conscious state and no evidence of the neurological deficit [11]. Cirrhosis patients with occurrence of upper or lower gastrointestinal bleeding could be diagnosed with acute gastrointestinal hemorrhage [12]. Bacterial infection was defined to include spontaneous bacterial peritonitis (SBP), pneumonia, cellulitis, urinary system infection and spontaneous bacteremia [13].

Definitions of organ failures were as follows [15]: the definition of liver failure was the total bilirubin (TBil) $\geq$ $12.0 \mathrm{mg} / \mathrm{dL}$; the definition of kidney failure was the serum creatinine $(\mathrm{Cr}) \geq 2.0 \mathrm{mg} / \mathrm{dL}$ or the use of renal replacement therapy; cerebral failure was defined by grade III-IV HE; the definition of coagulation failure was an international normalized ratio (INR) $\geq 2.5$; the definition of circulatory failure was the use of vasoconstrictors; the definition of respiratory failure was $\mathrm{PaO}_{2} / \mathrm{FiO}_{2} \leq 200$ or $\mathrm{SpO}_{2} / \mathrm{FiO}_{2} \leq 214$.

The MELD, CTP, SOFA and CLIF-C AD scores were calculated according to previously published criteria [5, 16-18]. All definitions and prognostic scores were applied at baseline.

\section{Treatment}

Standard medical therapies were used for all patients after diagnosis, such as bed rest, liver-protecting therapy, antiviral treatment, nutritional support, vitamins supplements, plasma and albumin transfusion, maintenance treatment of water-electrolyte and acid-base balance, control and prevention of complications.

\section{Clinical predictors}

Possible variables associated with short-term survival of $\mathrm{AD}$ of $\mathrm{CHB}$ cirrhosis were collected, including age; sex; mean arterial pressure; decompensation history; occurrences of organ failures or clinic complications; biochemical parameters, including alanine aminotransferase (ALT), aspartate aminotransferase (AST), $\gamma$-glutamyl transpeptidase (GGT), albumin (ALB), TBil, Cr, serum $\mathrm{Na}$, INR, white blood cell (WBC) count, absolute neutrophil count (NC), absolute platelet count (PLT), and HBV deoxyribonucleic acid (DNA) levels. Baseline data were obtained at the first diagnosis of $\mathrm{AD}$ of $\mathrm{CHB}$ cirrhosis. The 7-, 14- and 28-day survival rates were gained by searching the medical records.

\section{Statistical analysis}

Statistical analysise were conducted using SPSS version 20.0 (IBM, Armonk, NY, USA). Patient characteristics were compared by the Fisher's exact or $X^{2}$ tests (categorical variables) and the Mann-Whitney $U$ or $t$ test 
(continuous variables). Univariate risk factors that reached $p<0.05$ were subjected to Cox regression analysis.

The nomogram was built in terms of results of Cox regression analyses using $\mathrm{R}$ version 3.0.2 with the rms package [19]. According to the Akaike information criterion, backward step-down selection process was applied to the selection of final model [20]. The discrimination of the models were assessed in terms of the concordance index (C-index). Bootstraps with 1000 resamples were drawn to correct the $\mathrm{C}$-index. Comparisons between nomogram and other models were carried out by using the rcorrp.cens in the Hmisc package [21]. Calibration curves were also drawn to evaluate the concordance between predicted and observed probabilities. Decision curve analyses (DCA) were used to compare the benefits and improved performance of different models [22]. All tests were two-sided and were considered statistically significant when $p<0.05$.

\section{Results}

\section{Patient characteristics and outcomes}

In total, 509 and 620 patients from the derivation and external validation cohorts, respectively, were included for analyses. The baseline data of enrolled patients were summarized in Table 1 . In both sets, the average age was 5152 years, and the patients were predominantly men. 410 patients $(80.6 \%)$ were treated with nucleotide analogs (NUCs) and 99 patients (19.4\%) didn't received NUCs after hospital admission in the derivation cohort. 372 patients $(60.0 \%)$ were treated with NUCs and 248 patients (40.0\%) didn't receive antiviral therapy in the validation cohort. The most common complication was ascites, followed by HE (I-IV) and gastrointestinal hemorrhage at admission. Liver failure was the most frequent organ failure, which was followed by coagulation and cerebral failure.

When comparing the demographic and clinical characteristics between the derivation and validation sets, we found that patients in the derivation set had lower rates of previous decompensation and hyponatremia, and higher rates of complications (ascites, bacterial infection, gastrointestinal hemorrhage, $\mathrm{HE})$ at the time of admission $(p<0.05)$. Moreover, the validation set had lower $\mathrm{Na}$ levels and higher ALT, AST, GGT, TBil, Cr, INR, WBC, and HBV DNA levels $(p$ $<0.05)$. The validation set had higher 7-, 14- and 28-day risk of death than those of the derivation set $(p<0.05)$.

\section{Univariate and multivariate analyses}

Univariate analysis showed that age, male sex, previous decompensation, hyponatremia, ascites, bacterial infection, gastrointestinal hemorrhage, HE III-IV, SBP, hepatorenal syndrome, organ failure (liver, kidney, cerebral, coagulation, circulatory, and lung), AST, ALB, TBil, Cr, $\mathrm{Na}$, INR, WBC, and NC were significantly associated with poor prognosis of $\mathrm{AD}$ of $\mathrm{CHB}$ cirrhosis in the derivation set $(p<0.05$, Table 2$)$.
These variables were subjected to the Cox regression analyses. The results showed that only age $(\mathrm{HR}=1.057$, 95\% confidence interval [CI]: 1.032-1.082, $p<0.001$ ), previous decompensation $(\mathrm{HR}=2.449,95 \% \mathrm{CI}: 1.351-$ 4.438, $p=0.002)$, bacterial infection $(\mathrm{HR}=5.325,95 \% \mathrm{CI}$ : 3.015-9.405, $p<0.001)$, HE III-IV (HR $=4.660$, 95\% CI: 3.115-6.972, $p<0.001)$, and TBil $(\mathrm{HR}=1.053,95 \% \mathrm{CI}$ : $1.030-1.078, p<0.001)$ were independent risk factors for outcomes (Table 2).

\section{Derivation of the prognostic nomogram}

The nomogram was established on the basis of the coefficients gained from multivariate analysis, which included age, previous decompensation, bacterial infection, $\mathrm{HE}$, and TBil (Fig. 1). Each value of the factors was allocated the score in the point scale axis. By summing each single score and using that value in the total point scale axis, the total score could be easily calculated to assign the probability of survival for individual patients.

\section{Validation of the prognostic nomogram}

The C-index of the nomogram was 0.897 (95\% CI: 0.850 0.943 ) in the derivation cohort. To more effectively validate the practicability of the nomogram, we adopted an external cohort with $\mathrm{AD}$ of $\mathrm{CHB}$ cirrhosis for model validation. When the validation set was estimated by the established nomogram, the C-index was 0.839 (95\% CI: 0.811-0.867), suggesting that the nomogram is suitable for estimating the short-term outcome for $\mathrm{AD}$ of $\mathrm{CHB}$ cirrhosis.

The calibration curves were plotted showing that good agreements between the nomogram predictions and observed probabilities for the 7-, 14-, and 28-day outcomes in the primary (Figs. 2A-C) and external validation cohort (Figs. 2D-F).

\section{Performance of the nomogram compared with other models}

Futhermore, we compared the $\mathrm{C}$-indexes for evaluating the concordance of these models. The corrected C-index of our nomogram was 0.897 , which was significantly higher than that of the MELD (0.820), CTP (0.793), SOFA (0.868), and CLIF-C ADs (0.716) in the primary set $(p<0.001$, Table 3$)$. Meanwhile, the nomogram also had the highest $\mathrm{C}$-index (0.839) in the validation set, with statistical significance in comparison with MELD (0.826), CTP (0.741), SOFA (0.837), and CLIF-C ADs (0.569) $(p<0.001$ for all).

On DCA, our nomogram provided superior net benefit and improved performance for the 7-, 14-, and 28-day prognostic evaluation in the primary (Figs. 3A-C) and validation (Figs. 3D-F) cohorts relative to MELD, CTP, SOFA, and CLIF-C AD score models. This represents superior clinical usefulness of the nomogram over other score models. 
Table 1 Baseline characteristics in enrolled acute decompensation of CHB cirrhosis patients in the derivation and external validation cohort

\begin{tabular}{|c|c|c|c|}
\hline Characteristic & Derivation cohort $N=509$ & External validation cohort $N=620$ & $P$ value \\
\hline Age (yr) & $51.9 \pm 11.8$ & $51.4 \pm 11.3$ & 0.540 \\
\hline Male sex & $359(70.5)$ & $463(74.7)$ & 0.119 \\
\hline Mean arterial pressure (mm Hg) & $87.3 \pm 10.7$ & $87.3 \pm 12.2$ & 0.997 \\
\hline Previous decompensation & $197(38.7)$ & $328(52.9)$ & $<0.001$ \\
\hline \multicolumn{4}{|l|}{ Complication } \\
\hline Hyponatremia & $144(28.3)$ & $249(40.2)$ & $<0.001$ \\
\hline Ascites & $469(92.1)$ & $496(80.0)$ & $<0.001$ \\
\hline Bacteria infection & $106(20.8)$ & $75(12.1)$ & $<0.001$ \\
\hline Gastrointestinal hemorrhage & $123(24.2)$ & $62(10.0)$ & $<0.001$ \\
\hline Hepatic encephalopathy I-II & $100(19.6)$ & $46(7.4)$ & $<0.001$ \\
\hline Hepatic encephalopathy III-IV & $89(17.5)$ & $33(5.3)$ & $<0.001$ \\
\hline Spontaneous bacterial peritonitis & $21(4.1)$ & $19(3.1)$ & 0.337 \\
\hline Hepatorenal syndrome & $44(8.6)$ & $61(9.8)$ & 0.492 \\
\hline \multicolumn{4}{|l|}{ Organ failures } \\
\hline Liver & $96(18.9)$ & $152(24.5)$ & $<0.001$ \\
\hline Kidney & $23(4.5)$ & $64(10.3)$ & $<0.001$ \\
\hline Cerebral & $89(17.5)$ & $33(5.3)$ & $<0.001$ \\
\hline Coagulation & $56(11.0)$ & $120(19.4)$ & $<0.001$ \\
\hline Respiratory & $4(0.8)$ & $19(3.1)$ & 0.010 \\
\hline Circulation & $6(1.2)$ & $9(1.5)$ & 0.797 \\
\hline \multicolumn{4}{|l|}{ Biochemical parameters } \\
\hline Alanine aminotransferase $(U / L)$ & $130.7 \pm 388.4$ & $161.0 \pm 428.1$ & $<0.001$ \\
\hline Aspartate aminotransferase (U/L) & $125.4 \pm 211.3$ & $143.0 \pm 322.6$ & 0.005 \\
\hline Y-Glutamyltransferase (U/L) & $60.1 \pm 80.9$ & $66.5 \pm 86.9$ & 0.015 \\
\hline Albumin (g/L) & $29.5 \pm 16.3$ & $29.7 \pm 5.8$ & 0.764 \\
\hline Total bilirubin (mg/dL) & $6.8 \pm 9.3$ & $9.6 \pm 13.7$ & 0.004 \\
\hline Serum creatinine $(\mu \mathrm{mol} / \mathrm{L})$ & $80.3 \pm 58.1$ & $89.9 \pm 84.8$ & 0.016 \\
\hline Serum sodium (mmol/L) & $137.0 \pm 5.4$ & $134.6 \pm 6.8$ & $<0.001$ \\
\hline International normalized ratio & $1.7 \pm 1.5$ & $2.0 \pm 1.3$ & 0.001 \\
\hline White blood cell count $\left(\times 10^{9} / L\right)$ & $5.5 \pm 4.0$ & $6.4 \pm 4.8$ & 0.009 \\
\hline Neutrophil count $\left(\times 10^{9} / \mathrm{L}\right)$ & $4.1 \pm 5.8$ & - & - \\
\hline Platelet $\left(\times 10^{9} / \mathrm{L}\right)$ & $73.2 \pm 55.2$ & $79.1 \pm 59.2$ & 0.240 \\
\hline HBV-DNA (log copies/ml) & $4.1 \pm 1.7$ & $4.4 \pm 1.6$ & $<0.001$ \\
\hline \multicolumn{4}{|l|}{ Mortality } \\
\hline 7 days & $19(3.7)$ & $71(11.5)$ & $<0.001$ \\
\hline 14 days & $40(7.9)$ & 116(18.7) & $<0.001$ \\
\hline 28 days & $56(11.0)$ & $170(27.4)$ & $<0.001$ \\
\hline
\end{tabular}

Data are the mean \pm standard deviation for continuous valiables, and $\mathrm{n}(\%)$, frequency with percentage for categorical variables

\section{Discussion}

In the present study, a novel and easy-to-use nomogram was constructed to evaluate individual prognosis for $\mathrm{AD}$ of $\mathrm{CHB}$ cirrhosis. This nomogram demonstrated superior predictive capability and clinical usefulness relative to the current prognostic score models, including MELD, CTP, SOFA, and CLIF-C ADs.
In our derivation cohort, 11.0\% (56/509) of patients with $\mathrm{AD}$ of $\mathrm{CHB}$ cirrhosis died within 4 weeks-a result that was similar to that of another study [23]. A total of $13.0 \%$ (66/509) of the patients died within 90 days, and 56 of 66 patients died in the first 4 weeks; thus, we set up the 28-day statistical prognostic score model for patients with $\mathrm{AD}$ to find out those at the highest risk for death, which 
Table 2 Univariate and multivariate Cox regression analyses in patients with acute decompensation of CHB cirrhosis from the derivation cohort $(n=509)$

\begin{tabular}{|c|c|c|c|c|}
\hline & \multicolumn{2}{|l|}{ Univariate analysis } & \multicolumn{2}{|l|}{ Multivariate analysis } \\
\hline & $\mathrm{HR}(95 \% \mathrm{Cl})$ & $p$ value & $\mathrm{HR}(95 \% \mathrm{Cl})$ & $p$ value \\
\hline Age (yr) & $1.054(1.031-1.079)$ & $<0.001$ & $1.057(1.032-1.082)$ & $<0.001$ \\
\hline Male sex & $2.350(1.391-3.969)$ & $<0.001$ & & \\
\hline Mean arterial pressure $(\mathrm{mm} \mathrm{Hg})$ & $0.979(0.953-1.005)$ & 0.116 & & \\
\hline Previous decompensation & $2.419(1.420-4.120)$ & 0.001 & $2.449(1.351-4.438)$ & 0.002 \\
\hline \multicolumn{5}{|l|}{ Complication } \\
\hline Hyponatremia & $4.005(2.351-6.823)$ & $<0.001$ & & \\
\hline Ascites & $0.463(0.113-1.900)$ & $<0.001$ & & \\
\hline Bacteria infection & $2.479(1.443-4.259)$ & 0.001 & 5.325(3.015-9.405) & $<0.001$ \\
\hline Gastrointestinal hemorrhage & $2.301(1.042-5.081)$ & 0.039 & & \\
\hline Hepatic encephalopathy I-II & $1.426(0.439-4.631)$ & 0.555 & & \\
\hline Hepatic encephalopathy III-IV & $22.381(10.893-45.983)$ & $<0.001$ & $4.660(3.115-6.972)$ & $<0.001$ \\
\hline Spontaneous bacterial peritonitis & 4.392(1.988-9.703) & $<0.001$ & & \\
\hline Hepatorenal syndrome & 6.345(3.617-11.129) & $<0.001$ & & \\
\hline \multicolumn{5}{|l|}{ Organ failures } \\
\hline Liver & $2.720(1.583-4.672)$ & $<0.001$ & & \\
\hline Kidney & $4.839(2.370-9.881)$ & $<0.001$ & & \\
\hline Cerebral & 20.324(10.909-37.863) & $<0.001$ & & \\
\hline Coagulation & $9.389(5.547-16.891)$ & $<0.001$ & & \\
\hline Circulation & 10.148(3.657-28.166) & $<0.001$ & & \\
\hline Lung & $9.706(3.024-31.154)$ & $<0.001$ & & \\
\hline \multicolumn{5}{|l|}{ Biochemical parameters } \\
\hline Alanine aminotransferase $(\mathrm{U} / \mathrm{L})$ & $1.000(1.000-1.001)$ & 0.298 & & \\
\hline Aspartate aminotransferase $(\mathrm{U} / \mathrm{L})$ & $1.001(1.000-1.002)$ & 0.005 & & \\
\hline Y-Glutamyltransferase (U/L) & 0.999(0.996-1.003) & 0.722 & & \\
\hline Albumin (g/L) & $0.937(0.890-0.987)$ & 0.015 & & \\
\hline Total bilirubin (mg/dL) & $1.048(1.029-1.067)$ & $<0.001$ & $1.053(1.030-1.078)$ & $<0.001$ \\
\hline Serum creatinine $(\mu \mathrm{mol} / \mathrm{L})$ & $1.005(1.003-1.007)$ & $<0.001$ & & \\
\hline Serum sodium (mmol/L) & $0.904(0.872-0.937)$ & $<0.001$ & & \\
\hline International normalized ratio & $1.963(1.683-2.290)$ & $<0.001$ & & \\
\hline White blood cell count $\left(\times 10^{9} / \mathrm{L}\right)$ & $1.151(1.108-1.195)$ & $<0.001$ & & \\
\hline Neutrophil count $\left(\times 10^{9} / \mathrm{L}\right)$ & $1.175(1.128-1.225)$ & $<0.001$ & & \\
\hline Platelet $\left(\times 10^{9} / \mathrm{L}\right)$ & 0.998(0.993-1.004) & 0.576 & & \\
\hline HBV-DNA (log copies/ml) & $1.160(0.986-1.366)$ & 0.074 & & \\
\hline
\end{tabular}

would enable us to take effective treatment measures as soon as possible. In addition, most of studies on prognosis factors for liver decompensation cirrhosis did not strictly distinguish the etiology, such as viral hepatitis, alcohol, drugs, or other factors $[17,24]$. Our study only observed patients with $\mathrm{AD}$ of $\mathrm{CHB}$ cirrhosis as subjects; both blood biochemical tests and clinical complications were part of our prognosis factors. We also excluded the influence of liver transplantation, considering this has less application in our country. Moreover, to effectively evaluate the predictive ability of our nomogram versus those of established models, we adopted the external validation cohort to reduce the influence of drugs, treatments, and other factors on the results.

The proposed nomogram included age, two liver function indices (previous decompensation, TBil), and two complications (bacterial infection and HE). Many studies have suggested the correlation between age and prognosis in many diseases, and that old age is an important factor in poor prognosis for cirrhosis $[25,26]$. Recently, the CANONIC 

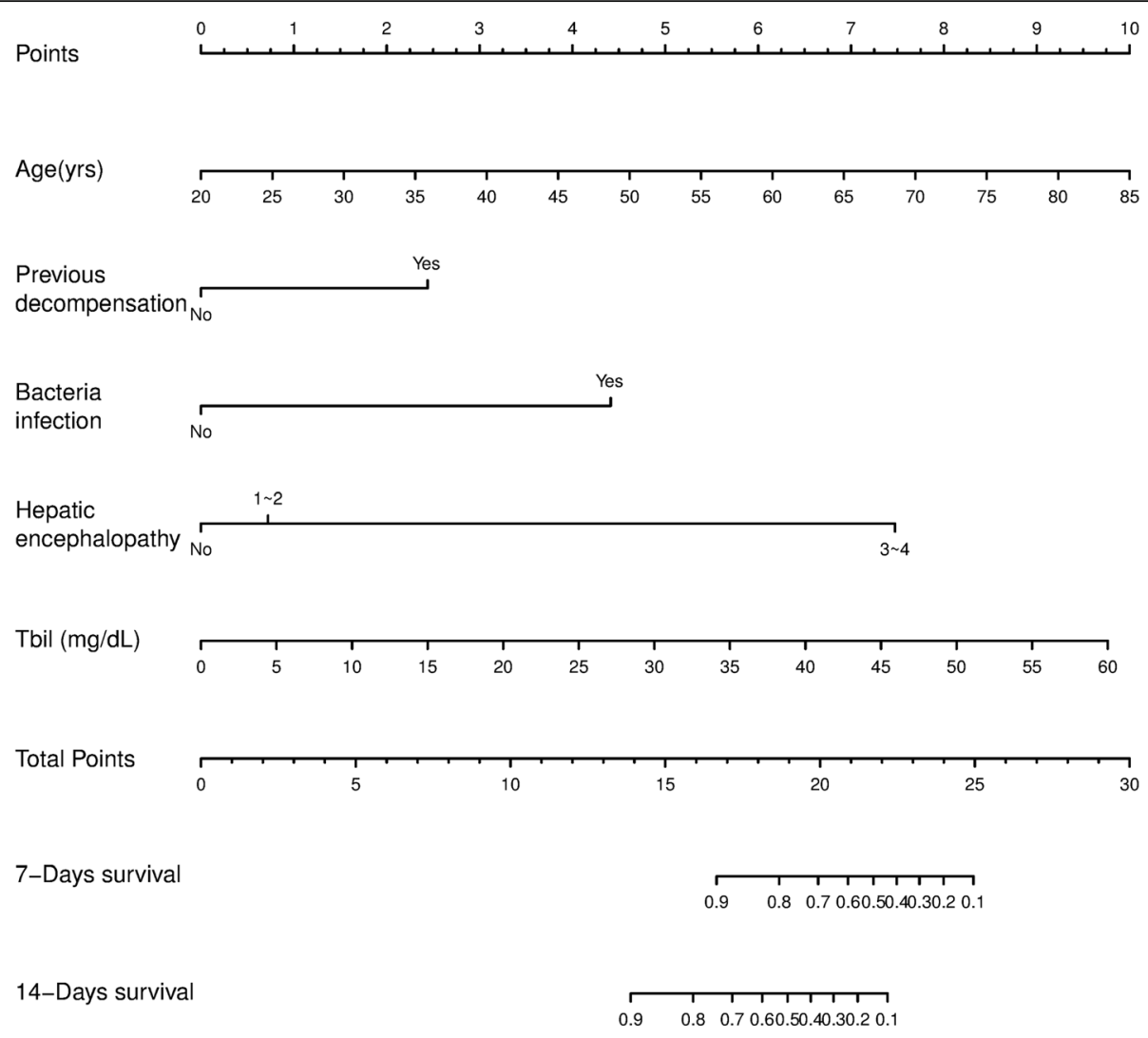

28-Days survival

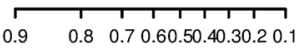

Fig. 1 Acute decompensation of CHB cirrhosis survival nomogram. To use the nomogram, the value of an individual patient is located on each variable axis, and a line is drawn upward to determine the number of points received for the value of each variable. The sum of these numbers is located on the total point axis, and a line is drawn downward to the survival axes to determine the likelihood of 7-, 14-, and 28-day survivals

study team built two models for ACLF (CLIF-C ACLFs) and $\mathrm{AD}$ (CLIF-C ADs) patients, where old age was considered an important indicator of poor outcomes $[5,15]$. This may be explained by loss of immune function or decline in tissue regeneration and repair [27, 28]. Serum TBil is commonly used as an indicator of the degree of liver damage and reserve function; it is also the main parameter of the CTP and MELD score systems [16, 17]. Previous decompensation could better reflect the underlying disease before the onset of AD. Bacterial infection represent the particularly important cause of liver failure and other complications [29]. The end-organ damaging effect of bacterial infection is more serious in liver cirrhosis and often culminates in newly developed liver and extrahepatic organ failures, which account for significant morbidity and mortality $[4,30]$. HE is a frequent and serious complication of cirrhosis $[31,32]$. Once HE occurs in patients with cirrhosis, the prognosis is poor, and liver transplant should be performed as soon as possible in order to increase the survival rate $[33,34]$.
In this study, the nomogram model performed well in predicting survival, as supported by the C-index ( 0.897 and 0.839 for the primary and validation cohorts respectively) and the calibration curve. Compared with MELD, CTP, SOFA, and CLIF-C ADs, the nomogram showed superior predictive capability for outcomes. Furthermore, the nomogram demonstrated better net benefit and improved performance for 7-, 14-, and 28-day prognostic evaluation in the derivation and validation cohorts compared with MELD, CTP, SOFA, and CLIF-C AD score models.

In current study, some patients didn't received antiviral therapy. One of the reasons is that patients with HE III-IV and gastrointestinal hemorrhage are unable to take drugs. Another reason is that the antiviral therapy was administered not only according to HBV replication levels, but also the willingness of the patient. So, considering the bad financial condition, many patients have to abandon the use of antiviral drugs. Besides, more patients didn't received the antiviral therapy in the validation cohort. The major reason is that the enrollment 


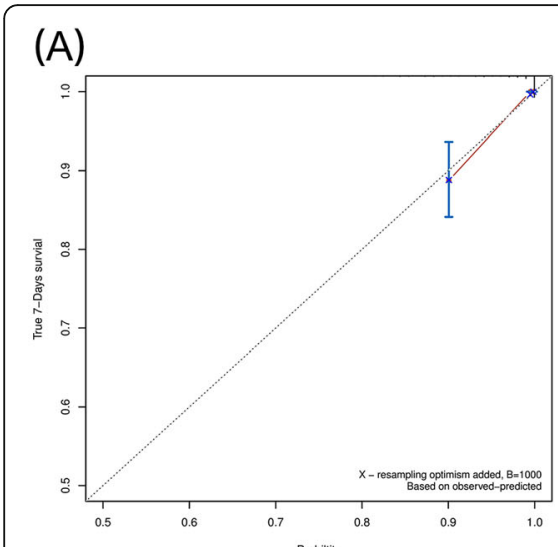

(B)

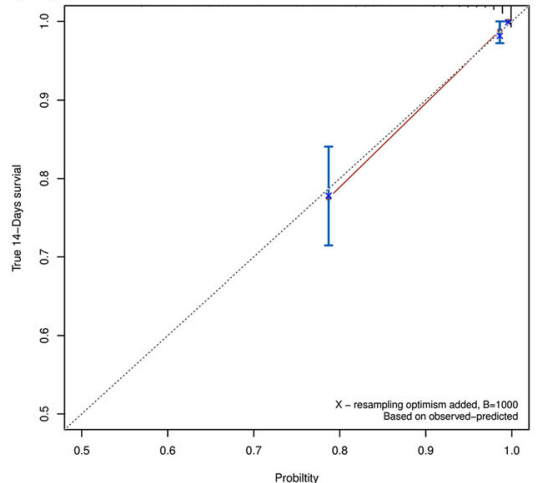

$(E)$
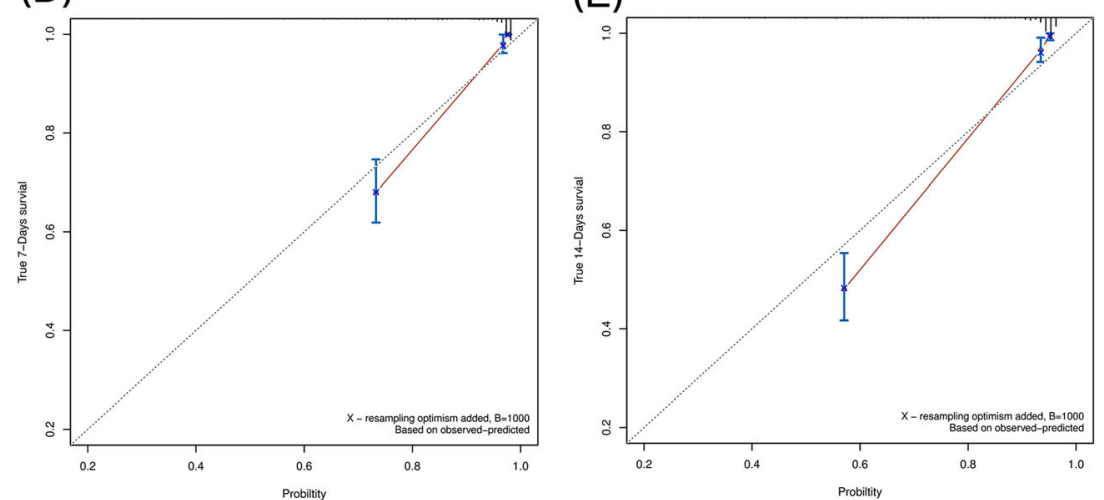

(C)

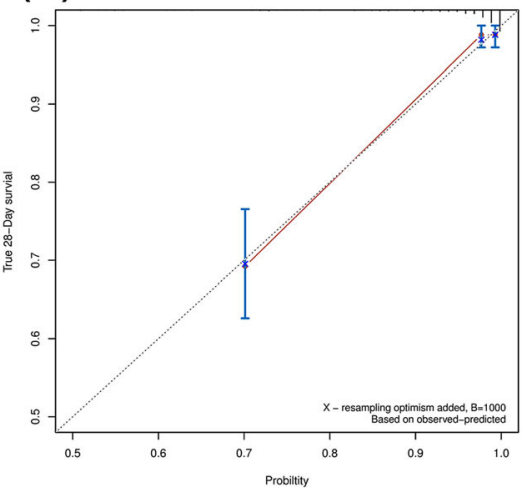

$(\mathrm{F})$

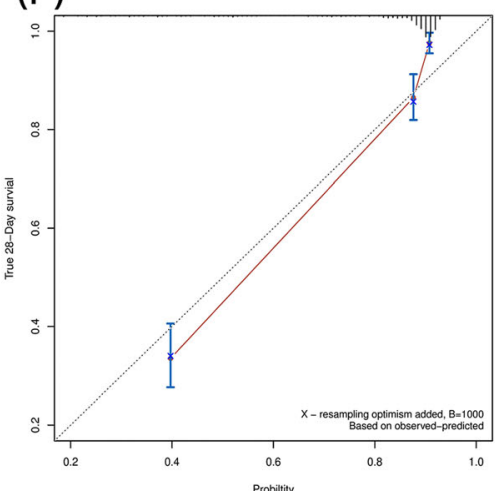

Fig. 2 The calibration curve of overall survival at 7, 14, and 28 days for the derivation cohort (a-c) and the external validation cohort (d-f). Nomogrampredicted probability of survival is plotted on the $x$-axis, and the actual survival is plotted on the $y$-axis. Dashed lines along the 45 -degree line through the point of origin represent the perfect calibration models in which the predicted probabilities are identical to the actual probabilities

Table 3 The predictive discrimination ability of the nomogram compared to the MELD, CTP, and SOFA score systems in the primary and validation cohorts

\begin{tabular}{|c|c|c|c|c|c|c|c|c|c|}
\hline & \multirow[t]{2}{*}{ C-index } & \multicolumn{2}{|c|}{ 95\% Cl for C-index } & \multicolumn{2}{|c|}{ Goodness of Fit } & \multicolumn{4}{|c|}{ Comparison of models } \\
\hline & & Lower & Upper & $\mathrm{LR}$ & $R^{2}$ & Dxy & SD & Z & $P$ value \\
\hline \multicolumn{10}{|c|}{ Primary cohort $(n=509)$} \\
\hline MELDs & 0.820 & 0.764 & 0.878 & 72.07 & 0.185 & -0.343 & 0.083 & -4.12 & $<0.001$ \\
\hline CTPs & 0.793 & 0.744 & 0.842 & 70.79 & 0.175 & -0.687 & 0.051 & -13.5 & $<0.001$ \\
\hline SOFAs & 0.868 & 0.829 & 0.907 & 119.46 & 0.282 & -0.398 & 0.075 & -5.33 & $<0.001$ \\
\hline CLIF-C ADs & 0.716 & 0.636 & 0.796 & 45.36 & 0.121 & -0.476 & 0.078 & -6.10 & $<0.001$ \\
\hline Nomogram & 0.897 & 0.850 & 0.943 & 165.63 & 0.374 & - & - & - & \\
\hline \multicolumn{10}{|c|}{ Validation cohort $(n=620)$} \\
\hline MELDs & 0.826 & 0.794 & 0.857 & 238.69 & 0.336 & 0.451 & 0.045 & 10.13 & $<0.001$ \\
\hline CTPS & 0.741 & 0.707 & 0.776 & 130.11 & 0.196 & -0.557 & 0.035 & -15.94 & $<0.001$ \\
\hline SOFAs & 0.837 & 0.807 & 0.866 & 255.24 & 0.349 & -0.215 & 0.049 & -4.39 & $<0.001$ \\
\hline CLIF-C ADs & 0.569 & 0.525 & 0.615 & 8.47 & 0.015 & -0.501 & 0.041 & -12.22 & $<0.001$ \\
\hline Nomogram & 0.839 & 0.811 & 0.867 & 268.38 & 0.363 & - & - & - & \\
\hline
\end{tabular}



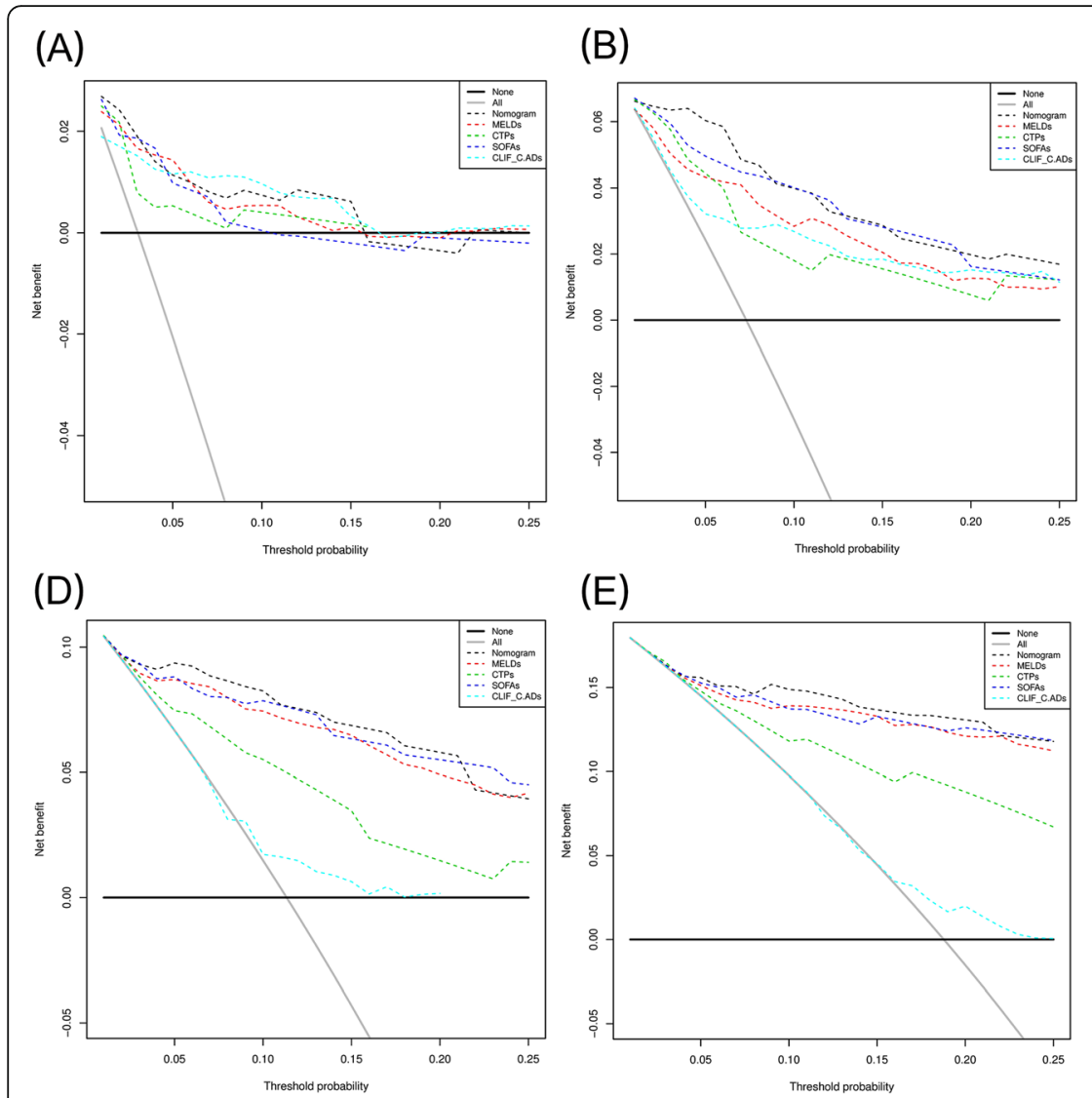

(B)

(E)

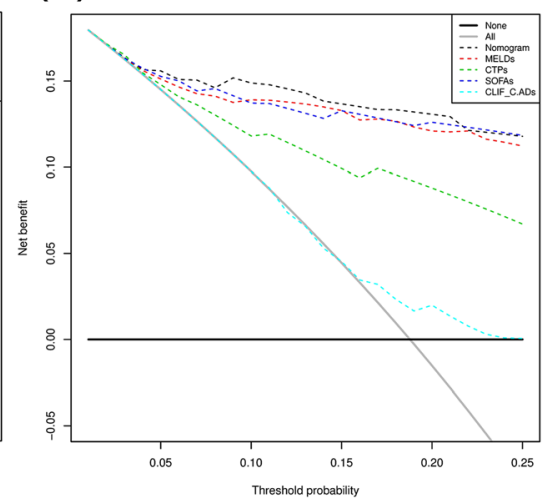

(C)

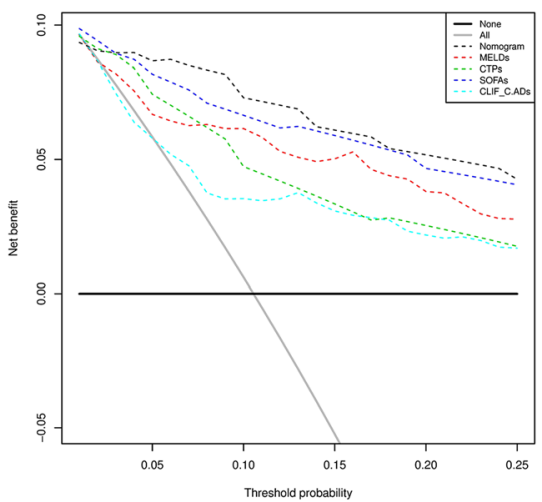

$(\mathrm{F})$

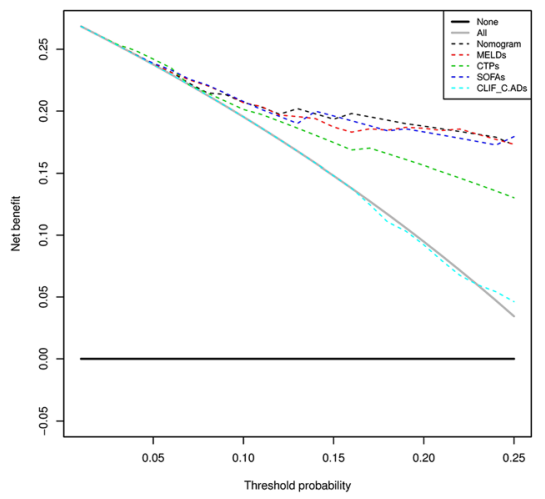

Fig. 3 Decision curve analysis at 7, 14, and 28 days for the derivation cohort (a-c) and the external validation cohort (d-f). Decision curve analyses depict the clinical net benefit in pairwise comparisons across the different models. The horizontal solid black line represents the assumption that no patients will experience the event, and the solid gray line represents the assumption that all patients will relapse. On decision curve analysis, the nomogram showed superior net benefit compared with other models across a range of threshold probabilities

time of patients in validation cohort is from January 2005 to December 2010, four years ahead of the derivation cohort. Antiviral drugs are more widespread with the development of healthcare in China. This reflects the clinical treatment situation of China objectively.

Our study has several limitations. First, our nomogram only included basic laboratory data. However, this study aimed to construct a reliable prognostic model. To avoid inevitable bias, subjective variables have not been included to construct our nomogram. Second, the nomogram was built on the basis of a retrospective cohort, and selection bias may exist. However, we have validated the model with data from another institution. The results consistently demonstrated the very good performance of our established nomogram.

\section{Conclusions}

To our knowledge, the nomogram model here is the first model developed to predict the individual prognosis of $\mathrm{AD}$ of cirrhosis in $\mathrm{CHB}$ patients to date. This provided better performance than MELD, CTP, SOFA, and CLIF-C AD scores, and it offers a foundation for individualized counseling and clinical treatment.

\section{Abbreviations}

AD: acute decompensation; ALB: albumin; ALP: alkaline phosphatase; ALT: alanine aminotransferase; AST: aspartate aminotransferase; CHB: chronic hepatitis B; Cls: confidence intervals; CLIF-C AD: Chronic Liver Failure Consortium Acute Decompensation; CTP: Child-TurcottePugh; DCA: decision curve analysis; GGT: $\gamma$-glutamyltranspeptidase; HBV: hepatitis B virus; HE: hepatic encephalopathy; HRs: hazard ratios; INR: international normalized ratio; MELD: Model for End-stage Liver Disease; NC: absolute neutrophil count; PLT: absolute platelet count; SOFA: Sequential Organ Failure Assessment; TBil: total bilirubin; WBC: white blood cell

\section{Acknowledgements}

We gratefully recognize the patients who participated in this study. We thank Yan Sang for her help with the data.

\section{Funding}

This study was supported by grants from the National Natural Science Foundation of China (No. 81473641) and the Beijing Municipal Administration of Hospitals Clinical Medicine Development of Special Funding Support (ZYLX201707). The funders had no role in study design, data collection and analysis, decision to publish, or preparation of the manuscript.

\section{Availability of data and materials}

The datasets generated and analysed during the current study are available from the corresponding author on reasonable request. 


\section{Authors' contributions}

XBW and HL were responsible for the conception and design of the study. FYG, XSL, GW, YXL, QZ, YL and HML were responsible for the acquisition and analysis of data. FYG, XSL and GW drafted the manuscript. All authors participated in interpretation of the findings. XBW revised and commented on the draft, and all authors read and approved the final version of the manuscript. All authors confirm that the content has not been published elsewhere and does not overlap with or duplicate their previously published work. All authors read and approved the final manuscript.

\section{Ethics approval and consent to participate}

The study protocol was in accordance with the ethical guidelines of the Declaration of Helsinki and was approved by the ethics committee of the Beijing Ditan Hospital (Beijing, China) and Renji Hospital, Shanghai Jiao Tong University. All patients provided written informed consent.

\section{Consent for publication}

Not applicable.

\section{Competing interests}

The authors declare that they have no conflicts of interest with regard to the publication of this research report.

\section{Publisher's Note}

Springer Nature remains neutral with regard to jurisdictional claims in published maps and institutional affiliations.

\section{Author details}

${ }^{1}$ Center of Integrative Medicine, Beijing Ditan Hospital, Capital Medical University, Beijing, China. ${ }^{2}$ Statistics Room, Beijing Ditan Hospital, Capital Medical University, Beijing, China. ${ }^{3}$ Department of Gastroenterology, Ren Ji Hospital, School of Medicine, Shanghai Jiao Tong University, Shanghai, China.

\section{Received: 24 August 2018 Accepted: 19 November 2018}

\section{Published online: 03 December 2018}

\section{References}

1. Dienstag JL, Ghany MG, Morgan TR, Di Bisceglie AM, Bonkovsky HL, Kim HY, et al. A prospective study of the rate of progression in compensated, histologically advanced chronic hepatitis C. Hepatology. 2011;54:396-405.

2. Jepsen P, Ott P, Andersen PK, Sørensen HT, Vilstrup H. Clinical course of alcoholic liver cirrhosis: a Danish population-based cohort study. Hepatology. 2010;51:1675-82.

3. D'Amico G, Garcia-Tsao G, Pagliaro L. Natural history and prognostic indicators of survival in cirrhosis: a systematic review of 118 studies. J Hepatol. 2006:44:217-31.

4. Moreau R, Jalan R, Gines P, Pavesi M, Angeli P, Cordoba J, et al. Acute-onchronic liver failure is a distinct syndrome that develops in patients with acute decompensation of cirrhosis. Gastroenterology. 2013;144:1426-37 1437.e1-9.

5. Jalan R, Pavesi M, Saliba F, Amorós A, Fernandez J, Holland-Fischer P, et al. The CLIF consortium acute decompensation score (CLIF-C ADs) for prognosis of hospitalised cirrhotic patients without acute-on-chronic liver failure. J Hepatol. 2015;62:831-40.

6. Touijer K, Scardino PT. Nomograms for staging, prognosis, and predicting treatment outcomes. Cancer. 2009;115:3107-11.

7. Kattan MW, Scardino PT. Evidence for the usefulness of nomograms. Nat Clin Pract Urol. 2007:4:638-9.

8. Wan G, Gao F, Chen J, Li Y, Geng M, Sun L, et al. Nomogram prediction of individual prognosis of patients with hepatocellular carcinoma. BMC Cancer. 2017;17:91.

9. Sternberg $\mathrm{CN}$. Are nomograms better than currently available stage groupings for bladder cancer. J Clin Oncol. 2006;24:3819-20.

10. Moore KP, Wong F, Gines P, Bernardi M, Ochs A, Salerno F, et al. The management of ascites in cirrhosis: report on the consensus conference of the international ascites Club. Hepatology. 2003;38:258-66.

11. Blei AT, Córdoba J. Hepatic encephalopathy. Am J Gastroenterol. 2001;96: 1968-76.

12. Garcia-Tsao G, Bosch J. Management of varices and variceal hemorrhage in cirrhosis. N Engl J Med. 2010;362:823-32.
13. Gustot T, Durand F, Lebrec D, Vincent JL, Moreau R. Severe sepsis in cirrhosis. Hepatology. 2009;50:2022-33.

14. Arvaniti V, D'Amico G, Fede G, Manousou P, Tsochatzis E, Pleguezuelo M, et al. Infections in patients with cirrhosis increase mortality four-fold and should be used in determining prognosis. Gastroenterology. 2010;139:124656 1256.e1-5.

15. Jalan R, Saliba F, Pavesi M, Amoros A, Moreau R, Ginès P, et al. Development and validation of a prognostic score to predict mortality in patients with acute-on-chronic liver failure. J Hepatol. 2014;61:1038-47.

16. Kamath PS, Kim WR. The model for end-stage liver disease (MELD). Hepatology. 2007:45:797-805.

17. Pugh RN, Murray-Lyon IM, Dawson JL, Pietroni MC, Williams R. Transection of the oesophagus for bleeding oesophageal varices. Br J Surg. 1973;60:646-9.

18. Vincent JL, Moreno R, Takala J, Willatts S, De Mendonça A, Bruining H, et al. The SOFA (Sepsis-related organ failure assessment) score to describe organ dysfunction/failure. On behalf of the working group on Sepsis-related problems of the European Society of Intensive Care Medicine. Intensive Care Med. 1996;22:707-10.

19. Frank E, Harrell J. Rms: Regression Modeling Strategies. R Package version 3. 4-0. https://CRAN.R-project.org/package=Hmisc.

20. Harrell FE Jr, Lee KL, Mark DB. Multivariable prognostic models: issues in developing models, evaluating assumptions and adequacy, and measuring and reducing errors. Stat Med. 1996;15:361-87.

21. Frank E, Harrell J. Harrell Miscellaneous. R Package version 3.9-2. https:// CRAN.R-project.org/package=Hmisc.

22. Vickers AJ, Elkin EB. Decision curve analysis: a novel method for evaluating prediction models. Med Decis Mak. 2006;26:565-74.

23. McPhail MJ, Shawcross DL, Abeles RD, Chang A, Patel V, Lee GH, et al. Increased survival for patients with cirrhosis and organ failure in liver intensive care and validation of the chronic liver failure-sequential organ failure scoring system. Clin Gastroenterol Hepatol. 2015;13:1353-60 e8.

24. Malinchoc M, Kamath PS, Gordon FD, Peine CJ, Rank J, ter Borg PC. A model to predict poor survival in patients undergoing transjugular intrahepatic portosystemic shunts. Hepatology. 2000;31:864-71.

25. Schiodt FV, Chung RT, Schilsky ML, Hay JE, Christensen E, Lee WM. Outcome of acute liver failure in the elderly. Liver Transpl. 2009;15:1481-7.

26. Faber W, Stockmann M, Schirmer C, Möllerarnd A, Denecke T, Bahra M, et al. Significant impact of patient age on outcome after liver resection for HCC in cirrhosis. Eur J Surg Oncol. 2014;40:208-13.

27. Zeeh J, Platt D. The aging liver: structural and functional changes and their consequences for drug treatment in old age. Gerontology. 2002;48:121-7.

28. Wynne HA, Cope LH, Mutch E, Rawlins MD, Woodhouse KW, James OF. The effect of age upon liver volume and apparent liver blood flow in healthy man. Hepatology. 1989;9:297-301.

29. Jalan R, Fernandez J, Wiest R, Schnabl B, Moreau R, Angeli P, et al. Bacterial infections in cirrhosis: a position statement based on the EASL special conference 2013. J Hepatol. 2014;60:1310-24.

30. Bajaj JS, O'Leary JG, Reddy KR, Wong F, Biggins SW, Patton H, et al. Survival in infection-related acuteon-chronic liver failure is defined by extrahepatic organ failures. Hepatology. 2014;60:250-6.

31. Ferenci P, Lockwood A, Mullen K, Tarter R, Weissenborn K, Blei AT. Hepatic encephalopathy-definition, nomenclature, diagnosis, and quantification: final report of the working party at the 11th world congresses of gastroenterology, Vienna, 1998. Hepatology. 2002;35:716-21.

32. Häussinger D, Schliess F. Pathogenetic mechanisms of hepatic encephalopathy. Gut. 2008;57:1156-65.

33. Orr JG, Morgan CL, Jenkins-Jones S, Hudson M, Conway P, Radwan A, et al. Resource use associated with hepatic encephalopathy in patients with liver disease. J Hepatol. 2014;60:S228-9.

34. Cordoba J, Ventura-Cots M, Simón-Talero M, Amorós À, Pavesi M, Vilstrup H, et al. Characteristics, risk factors, and mortality of cirrhotic patients hospitalized for hepatic encephalopathy with and without acute-on-chronic liver failure (ACLF). J Hepatol. 2014;60:275-81. 